\title{
Genet et la rhapsodie poétique
}

\author{
Élise Nottet-Chedeville
}

\section{(2) OpenEdition}

\section{Journals}

Édition électronique

URL : http://journals.openedition.org/genesis/3730

DOI : 10.4000/genesis.3730

ISSN : 2268-1590

\section{Éditeur :}

Presses universitaires de Paris Sorbonne (PUPS), Société internationale de génétique artistique littéraire et scientifique (SIGALES)

\section{Édition imprimée}

Date de publication : 15 décembre 2018

Pagination : 141-154

ISBN : 979-10-231-06282

ISSN : 1167-5101

\section{Référence électronique}

Élise Nottet-Chedeville, « Genet et la rhapsodie poétique », Genesis [En ligne], 47 | 2018, mis en ligne le 15 décembre 2019, consulté le 21 janvier 2021. URL : http://journals.openedition.org/genesis/3730 ; DOI : https://doi.org/10.4000/genesis.3730 


\title{
Genet et la rhapsodie poétique
}

\author{
Élise Nottet-Chedeville
}

$\mathrm{A}^{\mathrm{a} o}$ lors que la prose de Genet est souvent qualifiée de «poétique », l'auteur n'a publié que quelques poèmes entre 1942 et 1948 : «Le Condamné à mort», édité seul et à compte d'auteur en 1942; «Marche funèbre », édité avec le poème précédent sous le titre Chants secrets par Marc Barbezat en 1945 ; quelques poèmes publiés sans nom d'auteur sous le titre Vingt lithographies pour un livre que j'ai lu en $1945^{1}$; et enfin les poèmes «La Parade», «La Galère », «Un chant d'amour» et «Le Pêcheur du Suquet» édités avec les deux premiers poèmes de Genet sous le titre Poèmes par Marc Barbezat à L'Arbalète en 1948. Réédités depuis dans un même volume par les éditions Gallimard ${ }^{2}$, ces poèmes abordent des thématiques réprouvées par la société des années quarante comme celles du vol, du crime, de la pédérastie et de la pornographie, dans une langue emplie de préciosités et dans le cadre de vers réguliers tels l'alexandrin ou le décasyllabe aux rimes impeccablement plates, croisées ou embrassées. Ces «péchés de jeunesse ${ }^{3}$ », selon les propres mots de l'auteur, ne sont que peu étudiés ${ }^{4}$ alors qu'ils révèlent nombre de stylèmes de l'écrivain. Éparpillés, lacunaires et jamais analysés en tant que tels, les quelques documents composant l'avanttexte poétique de Genet apparaissent comme un matériau d'exception pour comprendre la naissance du style genétien et son mouvement, montrant de manière oblique ce qui se cache derrière le mythe élaboré par l'homme, la critique et la société. Parenthèse littéraire pour certains ou propédeutique indépassable pour d'autres, la production poétique de Genet doit donc aussi - et certainement d'abord - être étudiée dans une perspective génétique. Ainsi, à travers l'étude de documents d'archives et l'analyse de manuscrits de deux de ses poèmes - «La Parade» et «La Galère» - nous verrons que Genet n'a pas de conception figée ou sacralisée de l'écriture et qu'avec lui «rien ne se perd, rien ne se crée, tout se transforme» puisqu'il n'hésite pas à déplacer et à réutiliser vers, strophes et poèmes selon ses besoins, les desiderata d'autrui ou la recherche d'un style qui lui soit propre. Plus encore, par opportunisme, pragmatisme littéraire ou principe poétique, cette constance migratoire devient pour Genet un ouvroir potentiel de littérarité visible, fonctionnant sur le mode de la rhapsodie.

\section{Genèse du poème «La Parade»}

La genèse du poème «La Parade» offre un exemple de la pratique de ce Lavoisier poétique que se révèle être Genet et permet d'expliciter l'apparente complexité du poème et son hermétisme. Le dossier génétique valide par ailleurs

1. Cet ouvrage, illustré de lithographies de Roland Caillaux, n'a jamais été reconnu par Genet et n'est toujours pas référencé comme tel. D'après Albert Dichy, spécialiste de Genet, responsable du fonds Genet et directeur littéraire de l'Institut Mémoires de l'édition contemporaine, Genet ne voulut jamais reconnaître l'ouvrage car il trouvait les illustrations «vulgaires». Gallimard a choisi d'éditer les poèmes en question avec les autres poèmes de Genet en les regroupant sous le titre «poèmes retrouvés » - alors qu'ils n'ont jamais été perdus. On peut trouver l'ouvrage réédité en petit format : Vingt lithographies pour un livre que j'ai lu, comprenant des lithographies attribuées à Roland Caillaux, Cahiers Gai-Kitsch-Camp, Patrick Cardon (éd.), 1996.

2. Jean Genet, Le Condamné à mort et autres poèmes suivi du Funambule, Paris, Gallimard, coll. «Poésie», 1999

3. D’après Laurent Boyer, ami et exécuteur testamentaire de Genet, aujourd'hui décédé.

4. On recense quelques articles : Patrice Bougon, «Du désir des corps à l'amour des mots dans la poésie de Jean Genet : les voies paradoxales du détour», Modernités, n 36, textes réunis par Éric Benoît, Presses universitaires de Bordeaux, mai 2014; Pascal Caron, «Pour quelques paillettes en plus. Le Funambule de Jean Genet et l'image poétique du mouvement», Littérature, $\mathrm{n}^{\circ}$ 139, 2005; Bernard Delvaille, «La poésie comme salut», Le Magazine littéraire, ${ }^{\circ}$ 313, septembre 1993; Albert Dichy, «Jean Genet : la prison imaginaire », Le Magazine littéraire, $\mathrm{n}^{\circ} 247$, novembre 1987; Audrey Gilles, «La poésie du phallus dans Le Condamné à mort de Jean Genet», @nalyses, automne 2007; Ahmet Soysal, «Poésie de Jean Genet », Littera : Edebiyat Yazilari, vol. 10, 2001 ; quelques articles du Dictionnaire Jean Genet, Honoré Champion, 2014; ainsi qu'un mémoire de maîtrise : O. Martinaud, L'Érotisme dans les «Poèmes » de Jean Genet, sous la direction de Bernard Alazet, université de Paris III, 2001. 


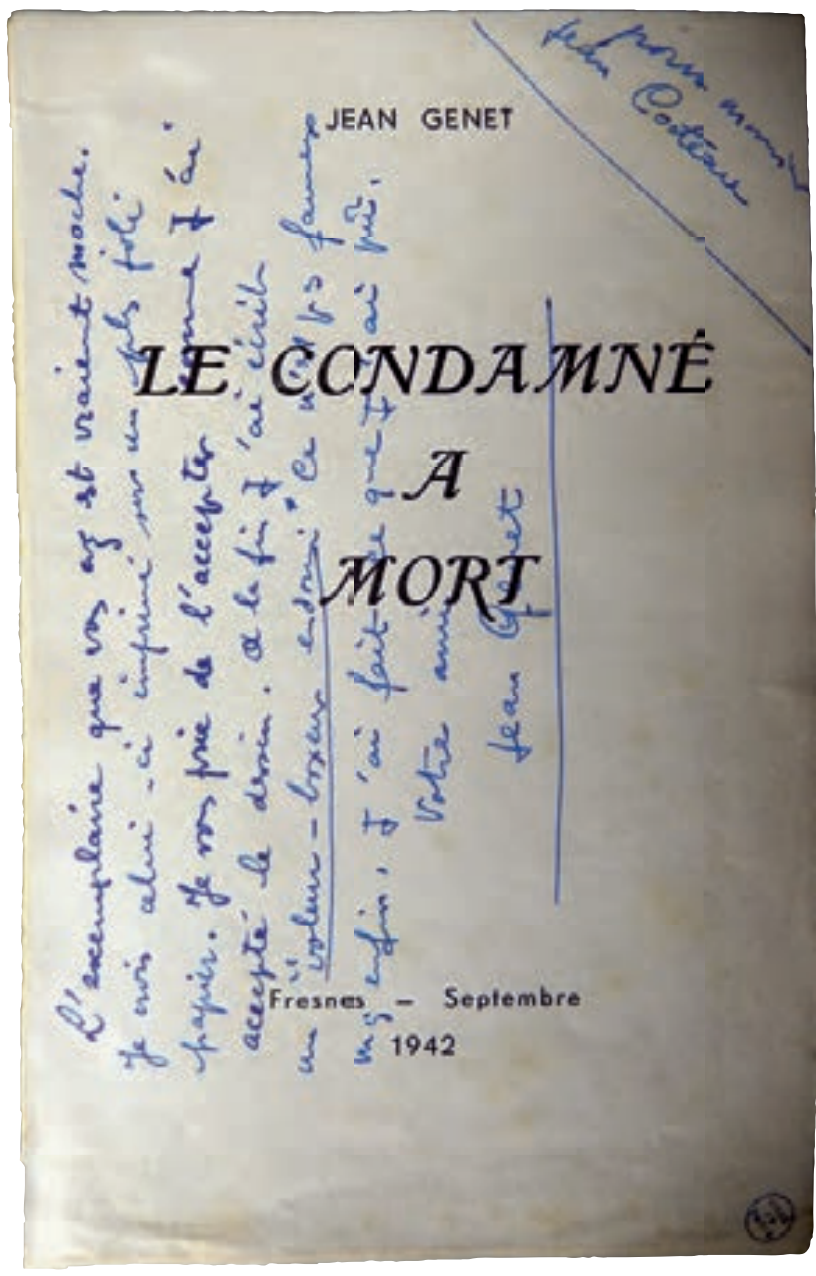

Fig. 1 : Dédicace de Genet inscrit sur l'exemplaire du Condamné à mort donné à Cocteau en février 1943.

«L'exemplaire que vous avez est vraiment moche. Je crois celui-ci imprimé sur un plus joli papier. Je vous prie de l'accepter comme j'ai accepté le dessin. À la fin j'ai écrit un "voleur-boxeur endormi ». Ce n'est pas fameux mais enfin, j'ai fait ce que j'ai pu. Votre ami Jean Genet».

(C) Bibliothèque historique de la Ville de Paris [MS-FS-05-7764]

définitivement la paternité des poèmes du recueil Vingt lithographies pour un livre que j'ai $l u^{5}$ et permet de dater assez précisément la rédaction des différentes strophes.

C'est le 10 février 1943 que le poète, alors en prison, envoie à son ami Roland Laudenbach un pneumatique au dos duquel il a écrit un poème, «Boxeur endormi », en l'honneur de Guy, l'un de ses amants 6 :

Au dos de cette carte, j'ai griffonné un petit poème à propos d'un jeune boxeur que j'appellais «Géo-la-voix-d'or-battu» et que j'ai gardé, en cellule, à la 230, bien des matins dans mes bras.

N'oubliez pas ce gosse. Vous savez, ce n'est pas un de mes gigolos. C'est un gamin très cher [?] $]^{7}$ : enfin vous verrez ${ }^{8}$.
Ledit poème est ensuite récité à Cocteau par Genet le 14 février lors de leur première entrevue, comme le rappelle Cocteau dans son Journal ${ }^{9}$. Lors de cette rencontre, Genet donne au «Prince des Poètes» un de ses exemplaires dédicacé du Condamné à mort (fig. 1) sur lequel est écrit le poème qu'il vient de réciter, intitulé cette fois «Voleur, Boxeur endormi » (fig. 2). Outre cette modification du titre, le poème comporte huit variantes et l'ajout d'un vers final par rapport à la version envoyée à Roland Laudenbach ${ }^{10}$.

Le 21 juillet 1943, de nouveau incarcéré, Genet envoie à son ami François Sentein un pneumatique dans lequel se trouve, au verso de l'unique feuillet, un poème, « À propos de la mort de Dubois qui buta le curé », composé de six strophes ${ }^{11}$, écrit en l'honneur d'Albert Dubois, condamné à mort pour l'assassinat de l'abbé Noël et exécuté dans la cour de la Santé le 16 juin 1943. Ledit poème est par ailleurs envoyé au même moment à François Bérard et Boris Kochno, amis de Genet et de Cocteau, sous le nom de «Vigile», avec quelques modifications. Il est accompagné de deux autres poèmes : «À Guy», composé de deux quatrains et d'un vers, et «Au même», composé de trois quatrains et d'un quintil12 (fig. 3).

En avril 1945, paraît le recueil Vingt lithographies pour un livre que j'ai lu, sans nom d'auteur, composé de vingt poèmes et vingt illustrations de Roland Caillaux. On y retrouve le poème «Voleur, Boxeur endormi » envoyé à Cocteau avec des variantes par rapport aux deux états précédents ainsi que le poème «À propos de la mort de Dubois qui buta le curé » ou «Vigile», scindé en deux, amputé de sa dernière strophe et largement modifié (fig. 4).

\footnotetext{
5. Cf. note 1 ci-dessus.
}

6. Ce poème est publié en fac-similé avec les lettres que Genet envoya à son ami François Sentein dans Lettres au petit Franz, Paris, Gallimard, coll. «Le Promeneur», 2000, p. 20.

7. Transcription incertaine de François Sentein.

8. Lettres au petit Franz, Paris, Gallimard, coll. «Le Promeneur», 2000, p. 20.

9. Jean Cocteau, Journal 1942-1945, Paris, Gallimard, coll. «La Pléiade», p. 269.

10. Cf. ci-dessous pour le détail de ces variantes.

11. Lettres au petit Franz, op. cit., p. 23, 91, 92 et 94.

12. Ces poèmes sont conservés à la Bibliothèque historique de la Ville de Paris avec le poème «La Galère » dans le fonds Boris Kochno. 


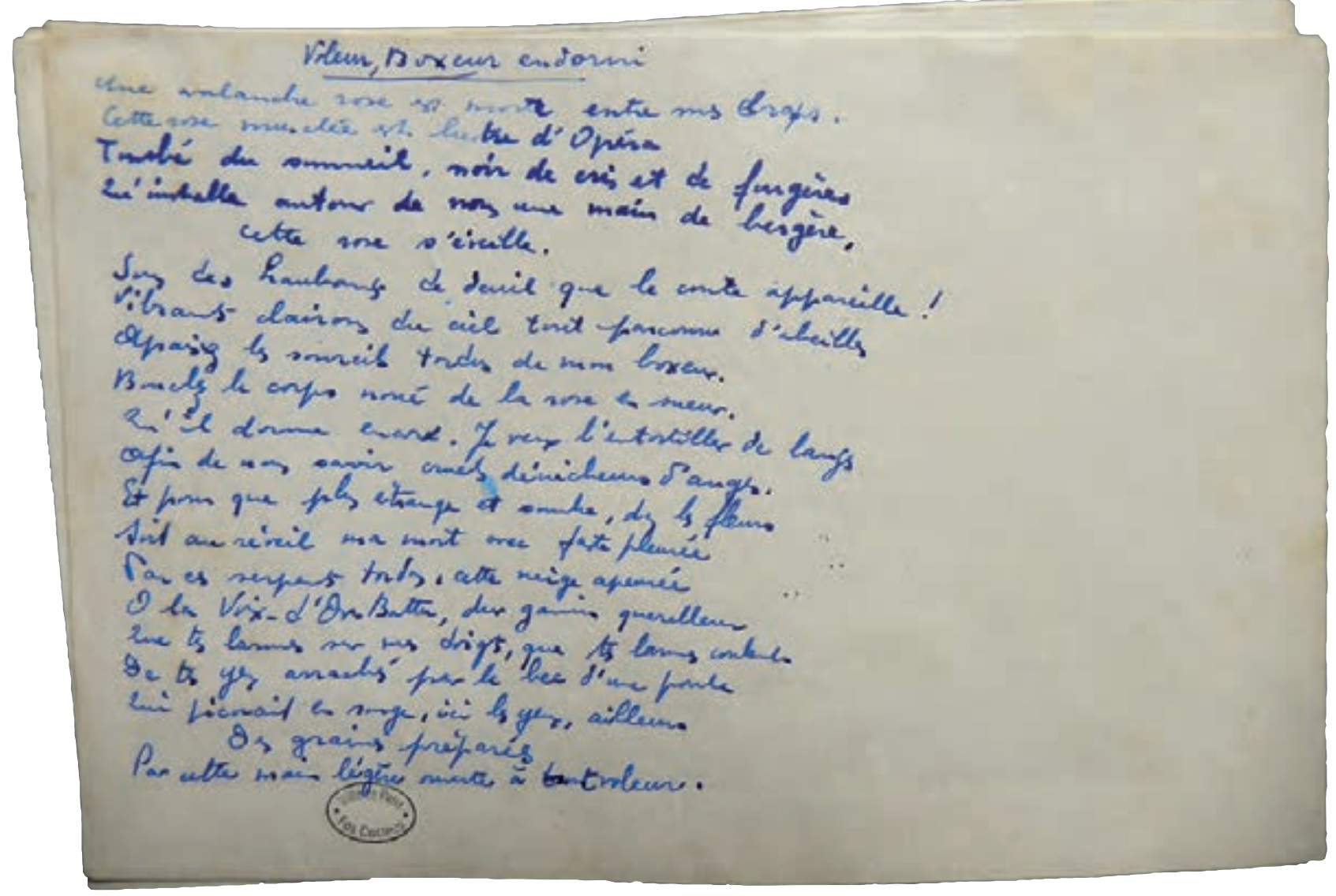

Fig. 2 : Poème manuscrit de Genet inscrit sur l'exemplaire du Condamné à mort donné à Cocteau en février 1943.

(C) Bibliothèque historique de la Ville de Paris [MS-FS-05-7764]

Genet travaille au même moment à l'élaboration d'une édition «complète» de ses poèmes avec Marc Barbezat, avec lequel il vient de publier son premier recueil poétique. Dès novembre 1945, il lui écrit : «Faites-moi parvenir les épreuves des poèmes. Et dites-moi la forme que vous donnerez au volume ${ }^{13}$. » Genet donne ensuite des consignes précises et n'hésite pas à dicter lui-même l'ordre de composition, comme l'indique cet échange entre les deux hommes, daté du 20 février 1946 :

Mon cher Ami,

Voici dans quel ordre vous placerez les poèmes. Ce sont là les seuls que je veuille garder. Le reste je m'en torche le cul.

Au début de «Un Chant d'Amour» vous mettrez cette dédicace : «À Lucien Sénémaud. »

Voici l'ordre du recueil général :

À M. Pilorge

Condamné à mort

Marche funèbre

À Lucien Sénemaud

Un Chant d'Amour

\section{La Galère}

Guy I et II

La Parade

Le flic

Secret

Voleur I et II

Boxeur

Et tout le reste vous regarde 14

Cet échange permet d'établir la genèse du recueil Poèmes 15 et celle du poème «La Parade». De fait, certains des titres mentionnés par Genet, à savoir «Guy I et II», «Le flic », «Secret», «Voleur I et II » et «Boxeur» n'apparaissent

13. Lettres à Olga et Marc Barbezat, Paris, Gallimard, coll. «L'Arbalète», 1988, p. 100.

14. Id., p. 110.

15. En ce qui concerne les variations de dédicace en particulier et la date de composition du «Pêcheur du Suquet» (poème qui n'apparaît pas dans cette lettre). 


\section{a $9 \%$}

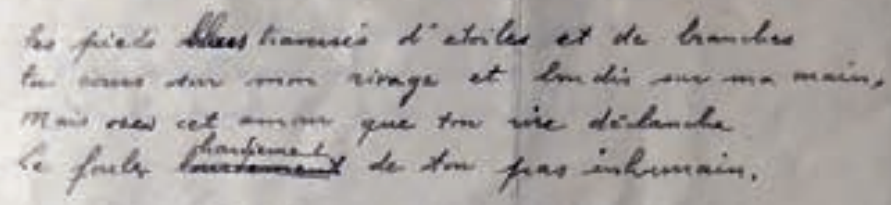

Fig. 3 : «À Guy» et «Au même», poèmes manuscrits de Genet, envoyés à Boris Kochno et Christian Bérard en 1943. ( ) Bibliothèque historique de la Ville de Paris, fonds Boris Kochno 
jamais tels quels dans les poèmes édités de Genet. Une étude attentive du recueil Poèmes en regard de cette lettre et du recueil Vingt lithographies pour un livre que j'ai lu illustré par Caillaux nous conduit à formuler l'hypothèse que ces noms de poèmes mentionnés par Genet correspondent pour certains à des poèmes envoyés à des proches - Cocteau, Laudenbach, Sentein, Bérard, Kochno - publiés dans Vingt lithographies pour un livre que j'ai lu en 1945, sans noms et sans termes en majuscules, et que lesdits poèmes furent repris et regroupés ensuite dans un ordre différent et sous forme de strophes comme un seul poème sous le titre général de «La Parade» dans le recueil Poèmes par Marc Barbezat en 1948.

Le relevé précis des variantes auquel nous nous livrons ci-dessous permet en outre de noter des différences de style entre chaque version, lesquelles sont imputables aux changements de destinataires et aux divergences de situations énonciatives : trivialité et intimisme dans la version envoyée au camarade François Sentein; érotisme affiché mais plus pudique et aspect archaïsant dans celle envoyée à Cocteau ; adoucissement voire euphémisation et généralisation dans celle réécrite pour Marc Barbezat. Genet écrivait et réécrivait en fonction de son interlocuteur et de son désir d'être publié.

Nous retrouvons ainsi les poèmes que Genet appelle «Guy I et II» dans sa lettre : ils correspondent aux trois premières «strophes » du poème «La Parade» commençant par «Silence, il faut veiller... ${ }^{16}$ » et aux deux strophes débutant par «On chante... ${ }^{17} »$. Le titre «Guy I et II» est corroboré par le contenu desdits poèmes et par la rencontre, en prison, avec l'amant nommé «Guy», dont l'importance est mise en exergue dans les manuscrits présentés. Les deux poèmes sont déjà présents dans le recueil Vingt lithographies pour un livre que j'ai lu de 1945, avec des variations, présentés séparément, accompagnés chacun d'une lithographie propre, et seule la lettre à l'initiale du vers est en majuscule ${ }^{18}$. Ils correspondent au poème envoyé à François Sentein, «À propos de la mort de Dubois qui buta le curé», et à celui envoyé à Bérard et Kochno sous le nom «Vigile», avec une réorganisation des vers des deux dernières strophes en une seule et de nombreuses variations. En voici la transcription dans l'encadré 1 (nous avons souligné les éléments textuels modifiés par Genet).

Le poème nommé ensuite par Genet dans sa lettre «La Parade» ne correspond, à strictement parler, qu'à la strophe en prose poétique dont les premiers mots sont «Dans la nuit du 17 au 18 juin, eut lieu, au camp de la Parade... ${ }^{19}$ » Il figure déjà dans Vingt lithographies pour un livre que j'ai $l u$, sans majuscule et sans italique, et ne comporte qu'une variante puisque «les ronces des chèvre-feuilles» est remplacé par «les ronces, les chèvre-feuilles ».

Le poème que Genet appelle «Le flic » correspond aux trois strophes débutant par «Esclaves d'un péché qui vous maintient en deuil / Vous tordez l'assassin...20» Dans le recueil Vingt lithographies pour un livre que j'ai lu, le poème ne comporte que deux quatrains, et non un quatrain et deux distiques, et deux vers assez différents (encadré 2).

Le poème intitulé «Secret» correspond aux cinq vers débutant par «Canaille oserez-vous... 21 » dans «La Parade» et reprend textuellement les cinq vers déjà présents dans Vingt lithographies pour un livre que j'ai lu.

Les poèmes nommés dans la lettre «Voleur I et II » correspondent quant à eux aux deux quatrains commençant par «Transparent voyageur...22 » dans «La Parade». Ils reprennent deux poèmes bien distincts dans Vingt lithographies pour un livre que j'ai lu, avec quelques variantes (encadré 3).

Enfin, le poème que Genet appelle «Boxeur» correspond aux strophes de «La Parade» qui vont de «Une avalanche rose est morte entre nos draps » à «tes souliers ». Il reprend en réalité un poème de Vingt lithographies pour un livre que j'ai lu ${ }^{23}$, réécriture du poème adressé à Cocteau, lequel était déjà une deuxième version de celui envoyé à Roland Laudenbach, auquel Genet a «cousu » l'autre poème envoyé en 1943 à Bérard et Kochno nommé «À Guy».

16. P. 59 dans Gallimard, coll. «Poésie» et p. 81 dans Vingt lithographies pour un livre que j'ai lu.

17. P. 60 dans Gallimard, coll. «Poésie», p. 85 dans Vingt lithographies pour un livre que j'ai lu.

18. Le procédé est le même pour les autres poèmes.

19. P. 61 dans Gallimard, coll. «Poésie», p. 65 dans Vingt lithographies pour un livre que j'ai lu.

20. P. 62 dans Gallimard, coll. «Poésie», p. 77 dans Vingt lithographies pour un livre que j'ai lu.

21. P. 63 dans Gallimard, coll. «Poésie», p. 21 dans Vingt lithographies pour un livre que j'ai lu.

22. P. 64 dans Gallimard, coll. «Poésie», p. 17 et 61 dans Vingt lithographies pour un livre que j'ai lu.

23. P. 65 et 66 dans Gallimard, coll. «Poésie», p. 37 et 69 dans Vingt lithographies pour un livre que j'ai lu. 


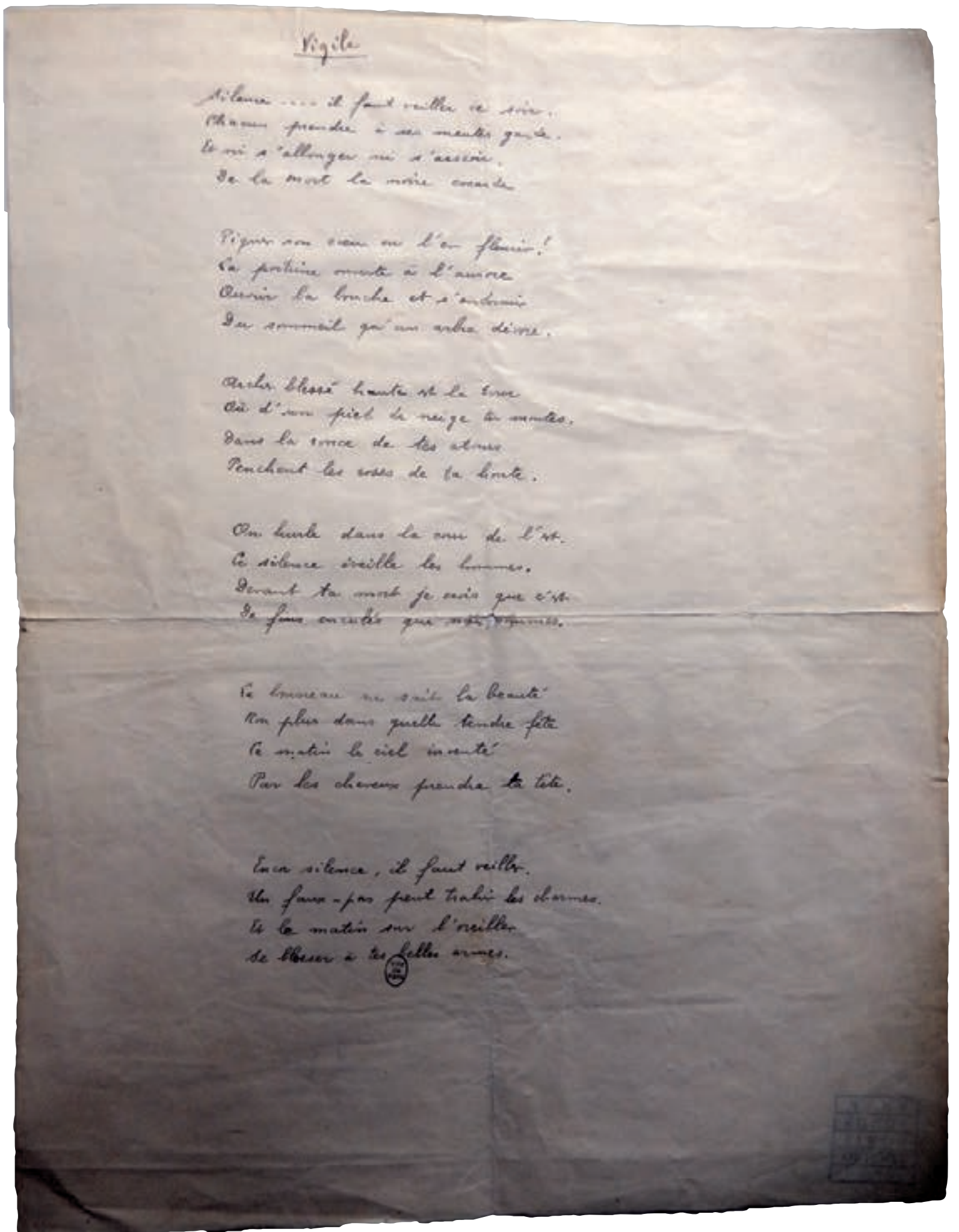

Fig. 4 : «Vigile», poème manuscrit de Genet envoyé à Boris Kochno et Christian Bérard en 1943. ๑) Bibliothèque historique de la Ville de Paris, fonds Boris Kochno 
Version de 1943

(«À propos de la mort de Dubois qui buta le curé») 1

Silence... il faut veiller ce soir. Chacun prendre à ses meutes garde. Et ni s'allonger ni s'asseoir De la Mort $^{2}$ la noire cocarde

Piquer son cœur ou l'en fleurir! La poitrine ouverte à l'aurore Ouvrir la bouche et s'endormir Du sommeil qu'un arbre dévore.

Archer blessé haute est la tour Où d'un pied de neige tu montes. Dans la ronce de tes atours Penchent les roses de ta honte.

On hurle dans la cour de l'est Le silence éveille les hommes. Devant ta [ill.] mort je crois que c'est De fiers enculés que nous sommes.

Le bourreau ne sait la beauté Non plus dans quelle tendre fête Ce matin, le Ciel éreinté ${ }^{3}$, Par les cheveux prendra ta tête,

Encor silence 4 il faut veiller, Un faux-pas peut trahir les charmes Et le matin sur l'oreiller Se blesser à ses 5 belles armes.
Version de 1945

(dans le recueil Vingt lithographies pour un livre que j'ai lu)

(«Guy I» et «Guy II», d'après Genet)

Silence, il faut veiller ce soir

Chacun prendre à ses meutes garde.

Et ne s'allonger ni s'asseoir

De la mort la noire cocarde

Piquer son cœur et l'en fleurir

D'un baiser que le sang colore.

Il faut veiller se retenir

Aux cordages clairs de l'aurore.

Enfant charmant, haute est la tour Où d'un pied de neige tu montes.

Dans la ronce de tes atours

Penches les roses de la honte.

On chante dans la cour de l'Est Le silence éveille les hommes.

Silence coupé d'ombre, et c'est

De fiers enculés que nous sommes.

Silence encor il faut veiller

Le Bourreau ignore la fête

Quand le ciel sur ton oreiller

Par les cheveux prendra ta tête.
Version de 1948

Encadré 1

(«La Parade»)

SILENCE, il faut veiller ce soir Chacun prendre à ses meutes garde. Et ne s'allonger ni s'asseoir De la mort la noire cocarde

Piquer son cœur et l'en fleurir D'un baiser que le sang colore Il faut veiller se retenir Aux cordages clairs de l'aurore.

Enfant charmant haut est la tour Où d'un pied de neige tu montes Dans la ronce de tes atours Penchent les roses de la honte.

ON CHANTE dans la cour de l'Est Le silence éveille les hommes. Silence coupé d'ombre et c'est De fiers enculés que nous sommes.

Silence encor il faut veiller Le Bourreau ignore la fête Quand le ciel sur ton oreiller Par les cheveux prendra ta tête.

1. Nommé «Vigile» par Genet quand il l'envoie à François Bérard et à Boris Kochno.

2. «mort» dans la version envoyée à Bérard et Kochno.

3. «ciel inventé» dans la version envoyée à Bérard et Kochno.

4. Une virgule dans la version envoyée à Bérard et Kochno.

5. «tes» dans la version envoyée à Bérard et Kochno.

Version de 1945

(dans le recueil Vingt lithographies pour un livre que j'ai lu)

(«Guy I» et «Guy II», d'après Genet)

Esclave d'un péché qui vous maintient en deuil Vous tordez l'assassin par ses poignets d'écume; Ses cris, ses crimes bleus égouttent dans votre œil L'encre qui vous révèle et de mort vous embrume.

Vous êtes les larrons, gardez le Fils de Dieu, Supputant du couteau et du sexe la forme. Or l'enfant, sur la paille allonge au fond des cieux, Ses chevilles de feuille afin qu'elles s'endorment.
Version de 1948

(«La Parade»)

ESCLAVES d'un péché qui vous maintient en deuil

Vous tordez l'assassin par mes poignets d'écume;

Ses cris, ses crimes bleus égouttent dans votre œil

L'encre qui vous révèle et de mort vous embrume.

Ô mes pâles larrons, gardez ce fils des dieux,

Qu'il crève! C'est sa mort votre noir uniforme.

Or l'enfant, sur la paille allonge au fond des cieux,

Ses chevilles de feuille afin qu'elles s'endorment.
Voleur I

TRANSPARENT voyageur des vitres du hallier Par la route du sang revenu dans ma bouche Les doigts chargés de lune et le pas éveillé. J'entends battre le soir endormi sur ma couche.
Voleur II

Encadré 3

VOTRE ÂME est de retour des confins de moi-même [«Bohême» en 1945] Prisonnière d'un ciel aux paresseux chemins

Où dormait simplement dans le creux d'un poème [ [ poëme» en 1945]

Une nuit de voleur sous le ciel de ma main. 
Version du 10 février 1943

(envoyée à R. Laudenbach)

«Boxeur endormi »

Une avalanche rose est morte entre mes draps.

Cette rose musclée est lustre d'Opéra

Tombé du sommeil, noir de cris et de fougères

Qu'installe autour de nous une main de bergère,

Cette rose s'éveille.

Sous des haubans de deuil que le conte appareille!

Vibrants clairons du ciel tout parcourus d'abeilles

Apaisez les sourcils tordus de mon boxeur.

Nouez le corps bouclé de la rose en sueur.

Qu'il dorme encore. Je vais l'entortiller de langes

Afin de nous sentir cruels dénicheurs d'anges.

Et pour que plus étrange et sombre, chez les fleurs

Soit, au réveil, ma mort avec faste pleurée

Par ces serpents tordus, cette neige apeurée

Ô la-Voix-d'Or-battu, mon doux mac querelleur

Que tes larmes sur mes doigts, que tes larmes coulent

De tes yeux arrachés par le bec d'une poule

Qui picorait en songe, ici les yeux, ailleurs

Des graines préparées

\section{Version de 1945}

(dans le recueil Vingt lithographies...)

Une avalanche rose est morte entre nos draps.

Cette rose musclée est lustre d'Opéra

Tombé du sommeil, noir de cris et de fougères

Qu'installe autour de nous une main de bergère,

Cette rose s'éveille

Sous les haubans de deuil que le conte appareille!

Apaisez les sourcils tordus de mon boxeur.

Bouclez le corps noué de la rose en sueur.

Qu'il dorme encor. Je veux l'entortiller de langes

Afin de nous savoir cruels dénicheurs d'anges

Et pour que plus étrange et sombre, chez les fleurs

Soit au réveil, ma mort avec faste pleurée

Par ces serpents tordus, cette neige apeurée.

Ô la voix d'or battu, dur gamin querelleur

Que tes larmes sur mes doigts que tes larmes coulent

De tes yeux arrachés par le bec d'une poule

Qui picorait en songe, ici les yeux, ailleurs

Des graines préparées

Par cette main légère ouverte à mon voleur.

Vibrants clairons du soir tout parcourus d'abeilles

1. «Sur» dans la version envoyée à Bérard.

2. «Le fouler lourdement hardiment » dans la version envoyée à Bérard.

3. «Des murs» dans la version envoyée à Bérard.

4. «Des miroirs » dans la version envoyée à Bérard.
Version du 14 février 1943

(écrite pour J. Cocteau)

«Voleur, Boxeur endormi »

Une avalanche rose est morte entre mes draps.

Cette rose musclée est lustre d'Opéra

Tombé du sommeil, noir de cris et de fougères

Qu'installe autour de nous une main de bergère,

cette rose s'éveille.

Sous des haubans de deuil que le conte appareille!

Vibrants clairons du ciel tout parcourus d'abeilles

Apaisez les sourcils tordus de mon boxeur.

Bouclez le corps noué de la rose en sueur

Qu'il dorme encore. Je veux l'entortiller de langes

Afin de nous savoir cruels dénicheurs d'anges.

Et pour que plus étrange et sombre, chez les fleurs

Soit au réveil ma mort avec faste pleurée

Par ces serpents tordus, cette neige apeurée.

Ô la voix-d'Or battu, dur gamin querelleur

Que tes larmes sur mes doigts, que tes larmes coulent

De tes yeux arrachés par le bec d'une poule

Qui picorait en songe, ici les yeux, ailleurs

Des graines préparées

Par cette main légère ouverte à tout voleur.

Version de 1948

(«La Parade»)

Une avalanche rose est morte entre nos draps.

Cette rose musclée ce lustre d'Opéra

Tombé du sommeil, noir de cris et de fougères

Qu'installe autour de nous une main de bergère,

Cette rose s'éveille!

Sous les haubans de deuil que le conte appareille!

Vibrants clairons du ciel tout parcourus d'abeilles

Apaisez les sourcils crispés de mon boxeur.

Bouclez le corps noué de la rose en sueur.

Qu'il dorme encor. Je veux l'entortiller de langes

Afin de nous savoir cruels dénicheurs d'anges

Et pour que plus étrange et sombre, chez les fleurs

Soit au réveil, ma mort avec faste pleurée

Par ces serpents tordus; cette neige apeurée.

Ô la voix d'or battu, dur gamin querelleur

Que tes larmes sur mes doigts que tes larmes coulent

De tes yeux arrachés par le bec d'une poule

Qui picorait en songe, ici les yeux, ailleurs

Des graines préparées

Par cette main légère ouverte à mon voleur.

Tes pieds bleus traversés d'étoiles et de branches

$\underline{\text { Tu cours sur mon rivage et bondis dans }}{ }^{1}$ ma main

Mais ose cet amour que ton rire déclenche

Hardiment le fouler ${ }^{2}$ de tes pieds inhumains!

$\underline{\text { Tu t'éveilles de } \text { moi }^{3}}{ }^{3}$ avec leur promptitude

$\underline{\text { Les spectres de mes dents }}{ }^{3}$, pour hanter l'escalier

$\underline{\text { Si rapide il faut donc Guy que ma solitude }}$

$\underline{\text { Par toi-même soit toi mon cœur multiplié }}$

Mais pour me parcourir enlève tes souliers. 
Nous proposons ci-contre (encadré 4) une transcription des quatre étapes du «poème»- ou plutôt de la «strophe»-en soulignant les modifications effectuées entre chaque étape.

Ces découvertes génétiques posent finalement question quant à l'interprétation que nous pouvons faire du poème «La Parade» : faut-il l'étudier comme une entité hétéroclite composée de plusieurs strophes, sachant qu'il est une réunion de divers poèmes publiés dans un tout autre ordre et écrits à différentes époques? Constitué par ajouts et réécritures de morceaux épars et différents dans leur contenu, leur forme et leur style, il nous invite par ailleurs à repenser le mouvement de genèse poétique de Genet sur le mode de la rhapsodie, c'est-à-dire comme un art de tisser et de coudre ensemble des pièces disparates, souvent liées sommairement et dont les jointures sont visibles. Cette pratique rhapsodique apparaît de manière encore plus nette dans la genèse du poème «La Galère ».

\section{Genèse du poème «La Galère »}

À propos de «La Galère », Genet écrit en 1943 : «Reçu une lettre de Denoël qui voudrait publier mon poème avec "autre chose". Je vais essayer de transformer "La Galère" et la lui envoyer 24. \ Cette information est corroborée par le témoignage de Jean-Paul Sartre qui rapporte des propos que Genet lui aurait tenus : «Vers ce temps-là, j’avais écrit deux poèmes sans qu'ils aient de rapport l'un avec l'autre. Je les ai mélangés, pensant donner plus d'obscurité, plus de densité à mes vers ${ }^{25}$. »

Le manuscrit du poème «La Galère », conservé à Austin, dédicacé et envoyé à Cocteau, présente un premier état très différent des quinze exemplaires manuscrits que Genet a recopiés ou fait recopier durant l'été 1944 par quelques acolytes. Beaucoup moins long que la version définitive 182 vers pour la version envoyée à Cocteau contre 273 pour la version finale -, il comprend des passages modifiés par la suite, des biffures, des réécritures, et un ordre des vers totalement différent de la version publiée. De nombreux vers n'apparaissent pas biffés mais seront réécrits dans la version publiée, ainsi que sur le manuscrit de «La Galère» daté de 1945 dont une photocopie est conservée à l'IMEC26. Ces premières observations accréditent le fait que le manuscrit envoyé à Cocteau est bien la première version de
«La Galère», qu'on peut certainement le dater de 1943, et que la version finale publiée est composée à partir de celui-ci et d'autres poèmes.

Par ailleurs, la Bibliothèque historique de la Ville de Paris conserve un deuxième manuscrit du poème, dédicacé à «Guy», qui est postérieur à celui envoyé à Cocteau puisqu'il contient les mêmes vers - dont certains sont modifiés ainsi que seize nouveaux vers. Contrairement aux autres exemplaires manuscrits du poème vendus aux enchères 27 qui ne peuvent tous être authentifiés, cette version, comme celle adressée à Cocteau, est bien de la main de Genet et apparaît donc comme l'avant-texte le plus sûr disponible actuellement. On trouve également dans ce fonds un premier tapuscrit reprenant le texte du manuscrit avec quelques variantes; il s'agit donc toujours de cette deuxième version. Figure enfin dans ce même fonds une troisième version carbone avec quatre-vingt-dix vers en plus, des modifications et plus aucune dédicace à Guy. Le texte du poème correspond à la version publiée en 1945 puis en 1947. Il s'agit donc de la troisième et dernière version du poème.

Nous reproduisons en figures 5 et 6 la première et la dernière page de l'exemplaire envoyé à Cocteau, et en figure 7 la première page du manuscrit détenu par la Bibliothèque historique de la Ville de Paris.

Il apparaît que le poème en alexandrins envoyé à Cocteau est beaucoup plus resserré, concentré et cohérent que la version publiée. Il progresse sur un mode épique à la gloire d'Harcamone, chef d'une galère imaginaire, sombrant dans les flots telle Ophélie, le cou entouré d'un lacet. Sa composition est rigoureuse : trois quatrains jouant le rôle de prologue et mettant en place le lien entre la prison et l'imaginaire de la galère, un vers se détachant introduisant la figure d'Harcamone, cent quarante-sept vers qui se suivent et content la geste d'Harcamone, un sizain déplorant la mort de

24. Lettres au petit Franz, op. cit., p. 88.

25. Jean-Paul Sartre, Saint Genet, comédien et martyr, Paris, Gallimard, coll. «NRF», 1952, p. 413

26. Genet avait le projet de l'éditer avec «Un chant d'amour» sous le titre Nouveaux poèmes de Jean Genet.

27. Il semblerait que Genet, comprenant la valeur pécuniaire qu'il pouvait tirer de la vente de ses manuscrits, ait recopié ou fait recopier une quinzaine de fois le manuscrit du poème «La Galère». C'est en tout cas le nombre d'exemplaires qui circulent dans les hôtels des ventes. 


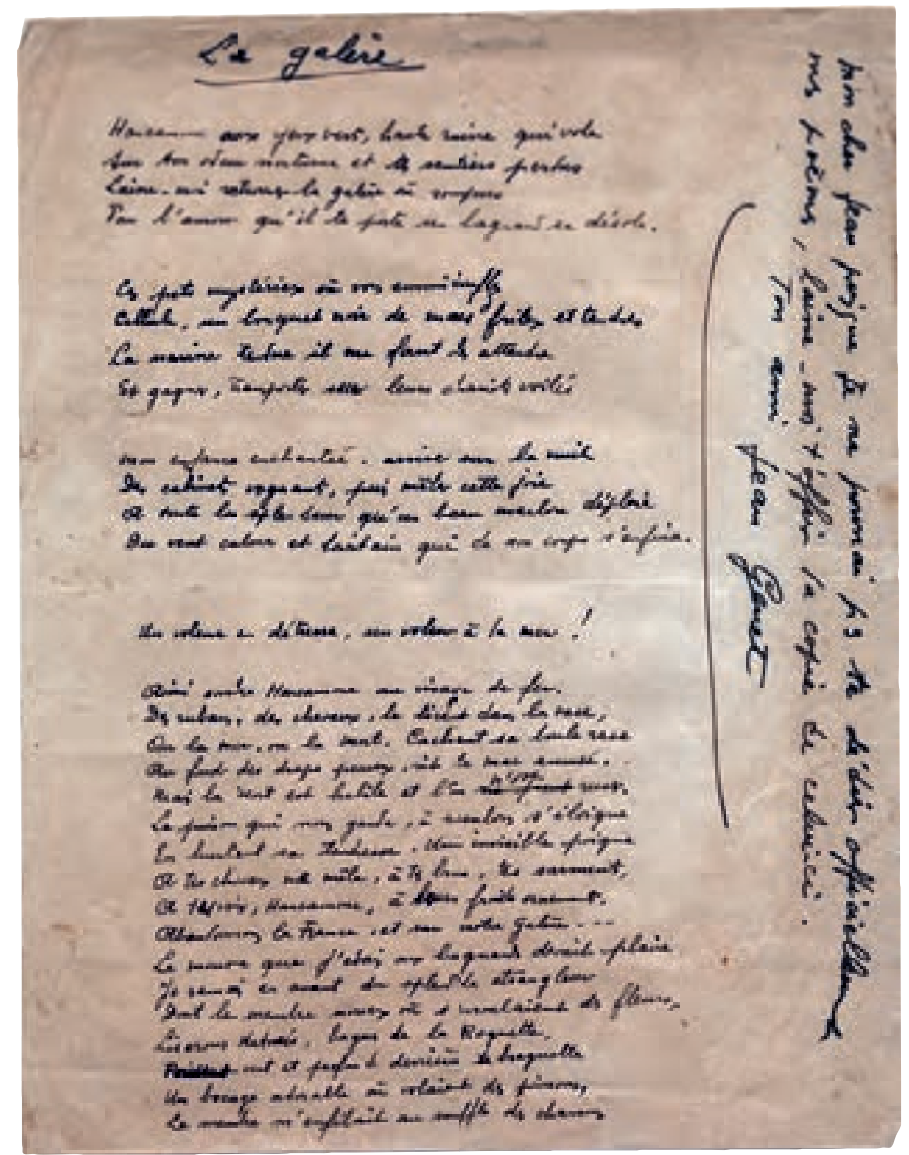

Fig. 5 : Première page du poème manuscrit «La Galère » envoyé à Cocteau en 1943.

(๑) Harry Ransom Center, Carlton Lake Collection, Austin

l'aimé, trois quatrains mettant en scène l'aura et la légende d'Harcamone qui perdure et un quatrain d'envoi 28 - mis en évidence par un point dessiné - qui revient à l'énonciateur et à sa solitude. Bien qu'Harcamone soit un des principaux personnages du Miracle de la Rose, les liens avec le roman sont extrêmement ténus.

Le poème connu et publié est en revanche beaucoup plus complexe et souvent qualifié d'hermétique et d'ésotérique. En regard de certaines lettres, de témoignages et des manuscrits, il apparaît que Genet a effectivement entremêlé non pas deux mais plusieurs poèmes pour composer le long poème final qu'est «La Galère». Le manuscrit conservé à la Bibliothèque historique de la Ville de Paris le confirme puisque les seize vers ajoutés à la première version semblent correspondre à deux courts poèmes, ajoutés à deux endroits différents au début du poème. Il semble que les quatre poèmes qu'évoque Jean Genet dans ses lettres à François Sentein les 16, 19 et 23 juin 1943 - à savoir «l'Enfant soudain», «Matelot I», «Matelot II» et «Prison» - aient été repris, entrecroisés et mêlés pour composer «La Galère » 29 . De fait, dans sa correspondance, le poète n'écrit plus rien

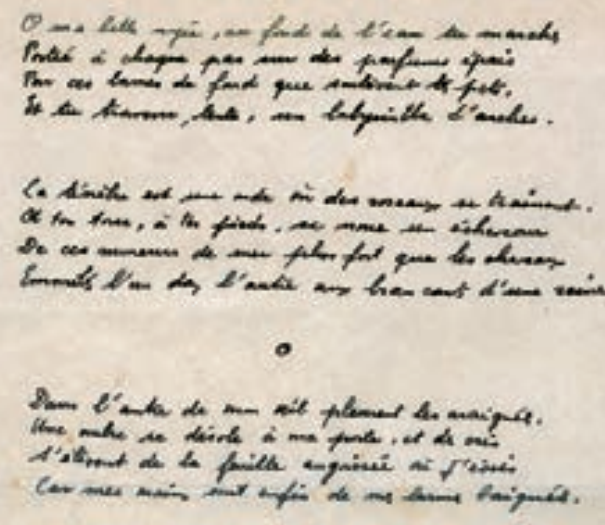

Fig. 6 : Dernière page du poème manuscrit «La Galère » envoyé à Cocteau en 1943.

(C) Harry Ransom Center, Carlton Lake Collection, Austin

sur ces poèmes à partir du moment où il fait référence à «La Galère». De même, dans les projets d'éditions ultérieures avec Marc Barbezat, il aborde d'autres poèmes sans plus jamais évoquer les quatre ci-dessus. Quant aux quelques poèmes présents dans le recueil illustré par Caillaux et non repris dans «La Parade», aucun ne semble répondre aux titres donnés par Genet.

Dans un premier temps, il est aisé de distinguer par un jeu de couleurs la version de Cocteau des vers ajoutés par la suite : nous reproduisons ci-dessous le palimpseste du début du poème publié en collection «Poésie Gallimard», en indiquant en rouge les vers initialement présents dans la version envoyée à Cocteau, en vert ceux ajoutés dans la version envoyée à Bérard et à Kochno, et en bleu les vers qui n'apparaissent que sur les manuscrits suivants et dans la version publiée.

28. Que nous retrouvons seul dans les poèmes publiés dans Vingt lithographies pour un livre que j'ai lu.

29. Lettres au petit Franz, Paris, Gallimard, coll. «Le Promeneur», 2000, p. 50,56 et 61 . 


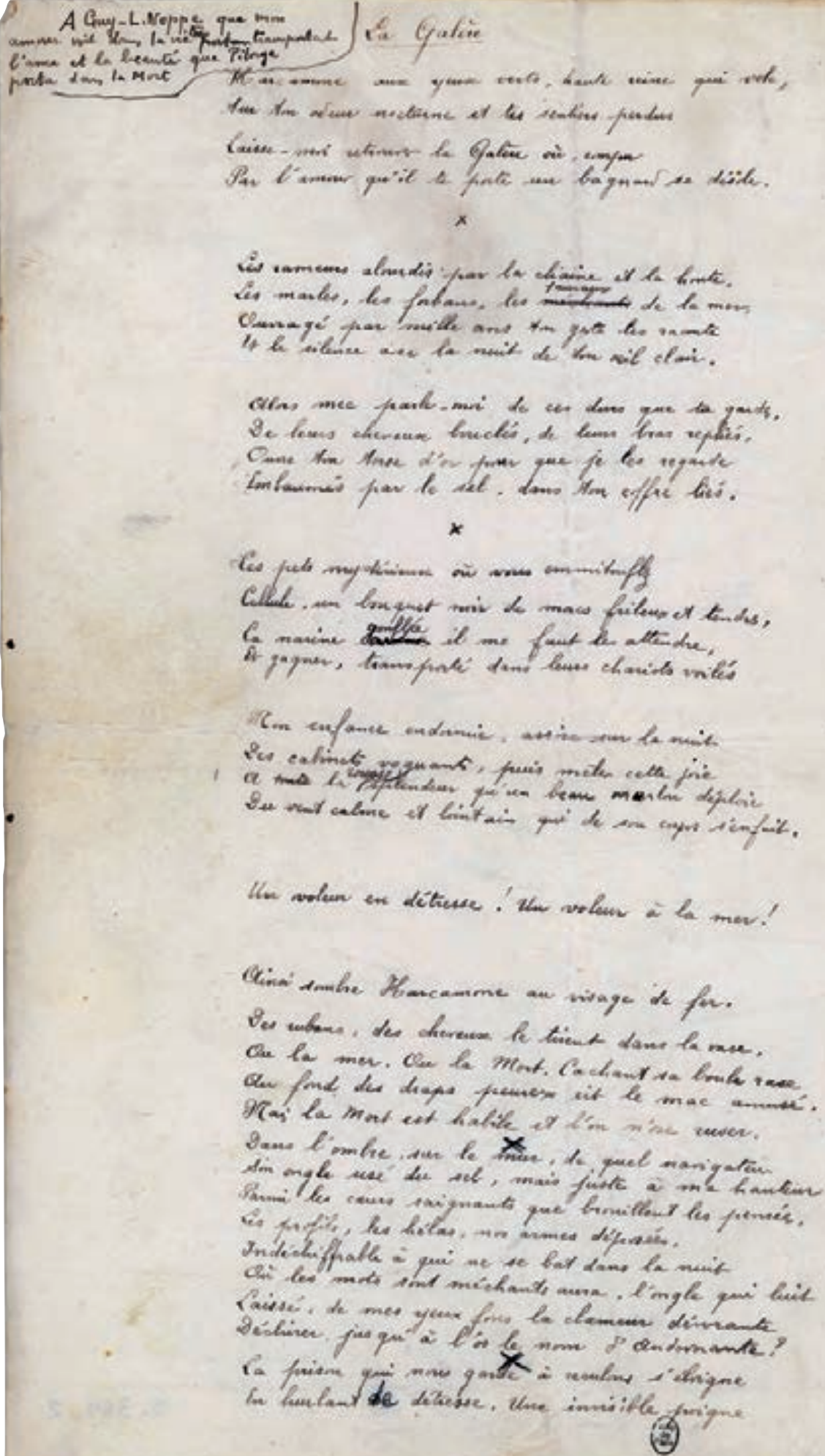

Fig. 7 : Première page du poème manuscrit «La Galère» envoyé à Boris Kochno et Christian Bérard en 1943. 
1UN FORÇAT2 délivré dur et féroce lance Un chiourme dans le pré 3 mais d'une fleur de lance 4 Le marlou Croix du Sud l'assassin Pôle ${ }^{5}$ Nord Aux oreilles d'un autre 6 ôtent ses ${ }^{7}$ boucles d'or. 8Les plus beaux sont fleuris d'étranges maladies9. Leur croupe de guitare éclate en mélodies. 10L'écume de la mer nous mouille11 de crachats. Sommes-nous remontés 12 des gorges d'un pacha?

On parle de me battre et j'écoute vos coups. Qui me roule Harcamone et dans vos plis me coud13 ?

Harcamone aux bras ${ }^{14}$ verts haute reine qui vole Sur ton odeur nocturne et les bois éveillés 15 16Par l'horreur de son nom ce bagnard endeuillé Sur ma galère chante et son chant me désole.

Les rameaux ${ }^{17}$ alourdis par la chaîne et la honte Les marles les forbans ces taureaux 18 de la mer Ouvragé par mille ans ton geste les raconte Et le silence avec la nuit de ton œil clair.

Les armes de ces nuits par les fils de la mort Portées mes bras cloués de vin l'azur qui sort De naseaux traversés par la rose égarée Où tremble sous la feuille une biche ${ }^{19}$ dorée... Je m'étonne et m'égare à poursuivre ton cours Étonnant fleuve d'eau des veines du discours20!

Empeste mon palais de ces durs que tu gardes 21 Dans tes cheveux bouclés sur deux bras repliés22 Ouvre ton torse d'or et que 23 je les regarde Embaumés par le sel dans 24 ton coffre liés.

Entr'ouverts ces cercueils ornés de fleurs mouillées Une lampe y demeure et veille mes noyées 25 .

26Fais un geste Harcamone allonge un peu ton bras 27 Montre-moi ce chemin par où tu t'enfuiras 28 Mais tu dors si 29 tu meurs et rejoins cette folle Où libres de 30 leurs fers les galériens s'envolent. Ils regagnent des ports titubants 31 de vins chauds Des prisons comme moi 32 de merveilleux cachots.

Ces pets mélodieux 33 où vous emmitouflez Cellule ${ }^{34}$ un bouquet vert ${ }^{35}$ de macs frileux et tendres La narine gonflée il faudra les attendre 36 Et gagner 37 transporté dans 38 leurs chariots voilés

Mon enfance posée à peine sur la nuit De papiers enflammés et mêler cette soie A la rousse splendeur qu'un grand marlou déploie39 Du vent calme et lointain qui de son corps s'enfuit.
1. Les huit vers suivants qui ouvrent le poème sont placés bien plus loin dans la version envoyée à Cocteau durant l'été 1943 et dans celle envoyée à Bérard. 2. Pas de majuscule dans la version envoyée à Cocteau, en 1945, dans l'édition originale de 1947 et en 1986.

3. Majuscule en 1945.

4. «Les chiourmes dans le Pré comme au fond d'une fosse» dans la version envoyée à Cocteau et dans celle envoyée à Bérard.

5. Un tiret en 1945 et dans l'édition originale de 1947.

6. «gâffe» dans la version envoyée à Cocteau.

7. «leurs» dans la version envoyée à Cocteau.

8. Les deux vers qui suivent sont inversés dans la version envoyée à Cocteau et dans celle envoyée à Bérard.

9. «Nos mâles sont fleuris» dans la version envoyée à Cocteau.

10. Les deux vers qui suivent sont inversés dans la version envoyée à Cocteau et dans celle envoyée à Bérard.

11. «mouillait» dans la version envoyée à Cocteau.

12. «Sommes-nous morts de Chine, esclaves de pachas» dans la version envoyée à Cocteau.

13. «couds» dans le projet d'édition de 1945.

14. «yeux» dans la version envoyée à Cocteau et celle envoyée à Bérard.

15. «sentiers perdus» dans la version envoyée à Cocteau et celle envoyée à Bérard.

16. «Laisse-moi retrouver la galère où rompus / Par l'amour qu'il te porte un bagnard se désole» dans la version envoyée à Cocteau et dans celle envoyée à Bérard, sans le «s » à «rompus ».

17. «Rameurs» dans la version envoyée à Bérard. Nous pensons que «rameaux» est une coquille.

18. «Les forbans» entre virgules et «méchants» biffé pour «taureaux» dans la version envoyée à Bérard.

19. «brèche» corrigée de façon manuscrite en «biche» dans le projet d'édition de 1945.

20. Un point dans l'édition originale de 1947.

21. «Alors mec parle-moi de ces durs que tu gardes» dans la version envoyée à Bérard.

22. «De leurs cheveux bouclés, de leurs bras repliés» dans la version envoyée à Bérard.

23. «Pour que» dans la version envoyée à Bérard.

24. «De» corrigée de façon manuscrite en «dans» en 1945.

25 . «Noyés» en 1945.

26. Les six vers suivants sont placés bien plus loin dans la version envoyée à Cocteau et dans celle envoyée à Bérard.

27. «Fais un geste, Harcamone, allonge un peu le bras» dans la version envoyée à Cocteau et dans celle envoyée à Bérard.

28. Un point dans la version envoyée à Cocteau, dans celle envoyée à Bérard et dans l'édition originale de 1947.

29. «Ou» dans la version envoyée à Cocteau, dans celle envoyée à Bérard en 1945 et en 1947.

30. «Dans» dans la version envoyée à Cocteau, dans celle envoyée à Bérard en 1945 et dans l'édition originale de 1947.

31. «Parfumés» dans la version envoyée à Cocteau et dans celle envoyée à Bérard. 32. «Comme ici» dans la version envoyée à Cocteau et dans celle envoyée à Bérard.

33. Les deux quatrains suivants sont placés bien avant dans la version envoyée à Cocteau et dans celle envoyée à Bérard ; «mystérieux » dans la version envoyée à Cocteau et dans celle envoyée à Bérard.

34. Virgule dans la version envoyée à Cocteau et dans celle envoyée à Bérard. 35. «Noir» dans la version envoyée à Cocteau et dans celle envoyée à Bérard. 36. «La narine tendue il faudra les attendre» dans la version envoyée à Cocteau et dans celle envoyée à Bérard; «tendue» biffée pour «gonflée» dans la version envoyée à Bérard.

37. Virgule dans la version envoyée à Cocteau et dans celle envoyée à Bérard.

38. «Sur» dans la version envoyée à Cocteau ; «dans» dans celle envoyée à Bérard.

39. «Mon enfance enchantée, assise sur la nuit / Des cabinets voguants, puis mêler cette joie / A toute la splendeur qu'un beau marlou déploie» dans la version envoyée à Cocteau et dans celle envoyée à Bérard avec «endormie» pour «enchantée » et «À la rousse splendeur » pour cette dernière. 
Nous constatons alors que les poèmes ainsi différenciés ne tissent pas les mêmes isotopies : celui envoyé à Cocteau est consacré à la geste d'Harcamone sur sa galère et comprend principalement des champs lexicaux liés à la mer, à la beauté et au sexe, tandis que le poème final tourne davantage autour de champs lexicaux liés aux marles et à l'enfermement pour certains vers et à la nature et aux fleurs pour d'autres. Archithème constant et structurant, la syllepse de la biche est utilisée à huit reprises dans le poème et structure un sous-ensemble. Elle est complètement absente dans le poème envoyé à Cocteau et est ajoutée à deux reprises dans les corrections de Genet effectuées dans le manuscrit de 1945 : deux vers du poème envoyé à Cocteau sont entièrement réécrits afin de faire figurer cette «biche» et d' assurer la transition - et la cohérence - avec les vers suivants.

Ces relevés prouvent que si les poèmes «Matelot $\mathrm{I}$ » et «Matelot II» visibles sur le manuscrit envoyé à Bérard et Kochno ont été ajoutés à «La Galère» envoyée à Cocteau, la version finale est un entremêlement avec le poème «Enfant soudain »- structuré autour de la syllepse de la biche et du poème «Prison», lui-même structuré autour du leitmotiv qui donnait son titre au poème. Ainsi, si nous reprenons uniquement le poème que nous pensons être anciennement «Enfant soudain », il apparaît que la lexie fait fonction, tout au long du poème, de syllepse structurante désignant tout à la fois l'animal et le jeune homme en fuite mais aussi le jeune garçon qui vient de perdre sa virginité. Cet éveil à la sensualité, ce jeu sexuel et cette naissance du jeune marle assurent la cohésion mais aussi la cohérence du poème ${ }^{30}$.

Le caractère textuel du poème n'est jamais apparu avec autant de clarté et n'a jamais pris autant de sens que pour ce dossier génétique de «La Galère » : Genet a bien tissé, cousu l'un à l'autre des pans de quatre poèmes distincts qui divergent stylistiquement. Le manque de cohérence et de cohésion textuelle que l'on attribue aujourd'hui au poème est une résultante de la genèse du poème, à savoir un mode d'écriture rhapsodique consistant à couper, mêler et réutiliser divers poèmes à d'autres fins. Et si le poème «La Galère» tire de la rhapsodie son mode de genèse, il gagne à être entendu et analysé comme une rhapsodie sur le plan générique, et non plus seulement génétique. L'entremêlement de strophes serait alors comme autant de pièces (mal) cousues de quatre poèmes épiques composés par le rhapsode Genet à la gloire d'amants divers, aimés et chantés.

Notre étude de la genèse des poèmes «La Parade» et «La Galère» nous fait écouter une musique caractéristique de Genet, celle de la rhapsodie. Ce mouvement de genèse poétique pourrait d'ailleurs s'appliquer à d'autres œuvres de Genet comme le Journal du Voleur, Notre-Dame-des-Fleurs ou Miracle de la Rose qui gagneraient à être analysées dans cette optique, plus poétique que romanesque. Certaines métalepses narratives apparaîtraient alors pour ce qu'elles sont avant tout : des résultantes d'un style de genèse, visibles à l'œil nu et explicables par les manuscrits. La GENETique textuelle promet d'ores et déjà de belles découvertes et offre un terrain en friche qui n'attend que d'être exploré.

30. Le sens de l'expression «se bicher» est d'ailleurs longuement expliqué par Genet dans Miracle de la Rose. 
ÉLISE NOTTET-CHEDEVILLE est agrégée de lettres modernes, ATER à Sorbonne Université et doctorante au sein de l'équipe d'accueil STIH (Sorbonne Université, EA 4509). Elle achève une thèse sur les poèmes de Jean Genet sous la direction de Jacques Dürrenmatt et s'intéresse plus largement à la génétique textuelle, à la stylistique et à la littérature des $\mathrm{XX}^{\mathrm{e}}$ et $\mathrm{XxI}^{\mathrm{e}}$ siècles.

elise_nottet@hotmail.com

\section{Genet et la rhapsodie poétique}

Peu étudié, l'avant-texte des Poèmes de Jean Genet révèle pourtant un mouvement de genèse spécifique. À travers l'analyse de documents d'archives et de manuscrits de deux de ses poèmes «La Parade» et «La Galère »- cet article met en lumière comment Genet conçoit l'écriture poétique sur le mode de la rhapsodie, n'hésitant pas à déplacer, à réutiliser, à renommer et à réécrire c'est-à-dire à coudre ensemble vers, strophes et poèmes selon ses besoins et les désirs d'autrui, et cela afin de plaire et de se faire éditer. Ce mouvement de migration textuelle aux coutures apparentes éclaire la genèse du recueil Poèmes, explique la discontinuité et le caractère hétéroclite, voire l'hermétisme de certains poèmes, et participe à la création d'un style propre.

The draft of Jean Genet's Poems has hardly ever been studied, yet it reveals a particular genesis. Through the analysis of archival documents and manuscripts of two of his poems -"The Parade" and "The Galley"- this article highlights Genet's conception of poetic writing as a rhapsody, with the poet often moving, reusing, renaming and rewriting -that is sewing together- verses, stanzas and poems according to his needs as well as other people's desires, with the aim of appealing to the reader and to be published. This textual migration, with visible seams, sheds new light on the genesis of Poems. It also explains the discontinuous, heterogenuous -and even hermetic- character of some poems, and participates in the creation of Genet's own style.

Der wenig erforschte Avant-Text der Poèmes von Jean Genet enthüllt in seiner Genese eine spezifische Bewegung. Anhand der Analyse von Archivdokumenten und Manuskripten von zweien seiner Gedichte - „La Parade“ und „La Galère“ - zeigt dieser Artikel, wie Genet das poetische Schreiben über den Modus der Rhapsodie konzipiert. Er zögert nicht, umzustellen, Elemente wiederzuverwenden, umzubenennen und neu zu schreiben - das heißt, Verse, Strophen und Gedichte nach seinen Bedürfnissen und nach den Wünschen anderer zusammenzunähen, und das, um zu gefallen und veröffentlicht zu werden. Diese Bewegung der Textmigration mit sichtbaren Nähten beleuchtet die Entstehung der Gedichtbandes Poèmes, erklärt die Diskontinuität und den uneinheitlichen Charakter, sogar die Hermetik bestimmter Gedichte und trägt zur Entstehung eines spezifischen Stils bei.
Poco estudiado, el pre-texto de los Poemas de Jean Genet pone sin embargo de manifiesto un movimiento de génesis específico. A través del análisis de documentos de archivo y de los manuscritos de dos de sus poemas -"La Parade" y "La Galère"-, este artículo muestra como Genet concibe la escritura poética según el modo de la rapsodia, sin dudar en desplazar, reutilizar, renombrar y reescribir -es decir, en coser juntos versos, estrofas y poemas-, de acuerdo a las necesidades y a los deseos de otra persona, con la finalidad de complacer y de hacerse editar. Este movimiento de migración textual, con costuras aparentes, esclarece la génesis de sus Poemas, explica la discontinuidad y el carácter heteróclito -y hasta el hermetismo de algunas de las composiciones- y participa en la creación de un estilo propio.

Pouco estudado, o ante-texto de Poèmes, de Jean Genet, revela uma dinâmica genética específica. Através da análise de documentos de arquivo e dos manuscritos de dois desses poemas «La Parade» e «La Galère»-, este artigo mostra como Genet concebe a escrita poética no modo de rapsódia, não hesitando em deslocar, reutilizar, renomear e reescrever, na verdade costurando juntos os versos, estrofes e poemas de acordo de acordo com a vontade de outros, para causar agrado e conseguir ser editado. Essa movimentação de textos com as costuras à mostra explica a génese da recolha, a sua descontinuidade, o carácter heteróclito, mesmo hermético, de alguns poemas e, em suma, é parte na criação de um estilo próprio.

Poco studiato, l'avantesto dei Poèmes di Jean Genet rivela pur tuttavia un movimento di genesi specifico. Attraverso l'analisi dei documenti d'archivio e dei manoscritti di due dei suoi poemi «La Parade»e «La Galère» - l'articolo mostra la concezione genettiana della scrittura poetica sul modo della rapsodia, che non esita a spostare, riutilizzare, rinominare, riscrivere, ossia a cucire insieme versi, strofe e poemi secondo i suoi bisogni e i desideri altrui, nell'intento di piacere e farsi pubblicare. Questo movimento di migrazione testuale dalle cuciture apparenti illumina la genesi della raccolta, spiega la discontinuità, il carattere eteroclito e perfino l'ermetismo di alcuni poemi, e partecipa alla creazione di uno stile proprio. 